\section{Testis size comparisons}

SIR-J.M. Diamond's thoughts on the difference in testis size between individuals of European and Chinese descent are accompanied by a pictorial representation in which the linear dimension is proportional to testis weight at autopsy. As proper scaling would have the linear dimension proportional to the cube root of weight, the size difference is vastly exaggerated.

Because drawings of human sex organs tend to catch the eye, casual perusers may have been left with the impression that pronounced testicular hypoplasia is the norm among males of Chinese heritage. Because sex-organ size is associated in the minds of some with courage, virility and the like, this careless illustration may initiate or promote a racial stereotype.

There has recently evolved into prominence a species of critic (for example, ref. 2) claiming that studies of intergroup differences are often hopelessly flawed. If their arguments have merit, it is important to avoid any hint of bias. I comment only because continuing interest in sex-organ size $^{3}$ may portend future articles. If so, I hope the results are presented in tabular form. If pictorial presentation is unavoidable, the figures should be pre-pared with due regard for proper organ-scaling to eliminate any hint of an ethnic slur.

Box 931, Brookline,

David C. Jolly

Massachusetts 02146, USA

1. Diamond, J.M. Nature 320, 488 (1986).

2. Gould, S.J. The Mismeasure of Man (Norton, New York, 1981).

3. Mittwoch, U. Nature 323, 117 (1986)

\section{More clues to gene rearrangements}

SIR-The demonstration by Möröy et al.' of rearrangement of the c-myc gene in hepatocellular carcinomas of hepatitis virus-infected woodchucks deserves further consideration because the nucleotide sequences surrounding the rearrangement exhibit several features reminiscent of those involved in the rearrangements of immunoglobulin genes mediated by recombinase enzymes ${ }^{2-4}$.

First, close to the breakpoint in the first intron of the woodchuck c-myc gene there is a heptamer-nonamer sequence motif which almost precisely matches that used by the recombinase; ; the heptamernonamer is 19 bases $5^{\prime}$ of the breakpoint rather than adjacent to it, as is usual, but these 19 bases may represent an $\mathrm{N}$ region ${ }^{6}$. Second, the rearrangement has occurred at a TG base pair in the c-myc intron, following an A residue on the juxtaposed DNA segment, consistent with the operation of an endonuclease, presumably associated with immunoglobulin recombinase activity, which cleaves at ${ }_{\mathrm{AC}}^{\mathrm{TC}}$ sequences? Thus, the breakpoint in this tumour shows characteristics similar to both physiologi- coding exons correspond to a part of the immunoglobulin or T-cell receptor loci. In contrast, these sequences hybridize with a 4.6 kilobase transcript, that is rather abundant in adult woodchuck liver, but absent from five other different woodchuck organs, including the spleen (unpublished results). Recombination of c-myc with cellular sequences specifically expressed in the liver argues for a localization of this event in liver cells.

We agree, however, with the hypothesis that the same enzymatic machinery might be involved in both hepatocellular carcinomas and lymphoid neoplams. The analysis of c-myc rearrangements in other hepatocellular carcinomas may clarify the analogies noted by Haluska and Croce.

TARIK MÖRÖY

Michelle Hadchouel tocyte that gives rise to the tumour, or it could be a result of hepatitis virus infection. We note that Hope et al. ${ }^{7}$ found the recombinase-like endonuclease not only in mouse fetal liver, a major site of B-cell lymphopoiesis, but also in adult liver.

Frank G. Haluska

The Wistar Institute,

36 th \& Spruce Streets,

Philadelphia, Pennsylvania 19104, USA

1. Möröy, T. et al. Nature 324, 276-279 (1986).

. Tsujimoto, Y. et al. Science 229, 1390-1393 (1985).

Haluska, F. G. et al. Nature 324, 158-161 (1986).

4. Finger, L.R. et al. Science 234, $982-984$ (1986)

5. Sakano, H. et al. Nature 286, 676-683 (1980).

6. Tonegawa, S. Nature 302, 575-581 (1983)

7. Hope, T.J.et al. Science 231, 1141-1145 (1986).

Möröy ET AL. REPLy - Haluska and Croce question whether the c-myc rearrangement could have taken place in infiltrating lymphocytes of neoplastic origin rather than in hepatocyte-derived tumour cells. Although Popper et al. have observed focal accumulation of haematopoietic cells in woodchuck liver tumours ${ }^{2}$ and we observe lymphoid cells and megacaryocytes in these tumours, several arguments suggest to us that the $c-m y c$ recombination took place in liver tumour cells.

First, in the DNA of tumour W64, the rearranged c-myc bands are almost as intense as the normal bands (by exon 2 hybridization), while in W93 DNA they are even stronger than the normal band (see ref. 1 , Figs $2 b$ and $3 b$ ). It seems unlikely that the small proportion of lymphoid cells to liver cells in these tumours could account for the relative intensity of rearranged and normal c- $m y c$ bands on Southern blots of tumour DNA. Second, rearranged c-myc bands could not be detected in Southern blots of non-tumorous liver DNA, which contains the same population of lymphocytes as the tumorous livers. In situ hybridization is now in progress to identify the cell type producing the observed high levels of $c-m y c$ transcripts and therefore carrying a mutated c-myc allele. Finally, we have no evidence so far that the s/bg sequences ${ }^{1}$ adjacent to c-myc \section{Pierre Tiollais \\ Marie-ANNICK BUENDiA}

Institut Pasteur,

28, rue du Dr Roux,

75724 Paris Cedex 15, France

1. Möröy, T. et al. Nature 324, 276-279 (1986)

2. Popper, H. et al. Hepatology 1,91-98 (1981).

\section{Electronic structure of the benzene molecule}

SiR-It is gratifying to me that a recent spin-coupled valence-bond treatment of the benzene molecule by Cooper, et al. should have led the authors to conclude that the Kekule description of the molecule, as expressed in the classical VB form, is much closer to reality than a description in terms of delocalized molecular orbitals.

The earliest reference given by Cooper et al. is 1961. In 1933, however, I published a simple graphical method of evaluating the matrix elements of the Rumer structures of molecules ${ }^{2}$ and applied it to the five structures of benzene:
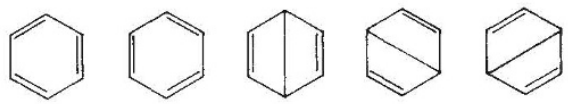

Solution of the secular equation ${ }^{3}$ gives the ratio of the occupation numbers of the Kekule structures and the other three as $0.3898: 0.0735$, close to the values 0.4028 : 0.0648 obtained by Cooper et al., who state that "They [their values] are astonishingly close to the values given long ago by Coulson ". Coulson, however, had in 1961 reproduced our 1933 calculation; the astonishingly close values had in fact been obtained in the earliest years of valencebond quantum mechanics.

\section{Linus Pauling Institute}

Linus PAULing

of Science and Medicine,

440 Page Mill Road,

Palo Alto, California 94306, USA

1. Cooper, D.L., Gerratt, J. \& Raimondi, M. Nature 323, 699 (1986)

Pauling, L. J. chem. Phys. 1, 280 (1933)

Pauling, L. \& Wheland, G.W J. chem. Phys. 1.362 (1933)

. Coulson, C.A. Valence 2nd edn, 249 (Clarendon, Oxford, 1961). 